\title{
Nucleation Control in Physical Vapor Transport Growth of AIN Single Crystals on Polycrystal Tungsten Substrates
}

\author{
Honglei WU*, Zuoyan QIN, Xueyong TIAN, Zhenhua SUN, Baikui LI, Ruisheng ZHENG, \\ Ke WANG
}

Key Laboratory of Optoelectronic Devices and Systems of Ministry of Education and Guangdong Province, College of
Optoelectronic Engineering, Shenzhen University, Shenzhen 518060, China

cross ref http://dx.doi.org/10.5755/j01.ms.26.2.21575

Received 04 September 2018; accepted 10 November 2018

\begin{abstract}
The improved resistively-heated furnace with two heaters established a vertical thermal gradient to control nucleation during AlN single crystals Physical Vapor Transport (PVT) growth on polycrystal tungsten substrates. During the high temperature $\left(>1850{ }^{\circ} \mathrm{C}\right)$ heating process, the reverse temperature field (i.e., the temperature difference between the sublimation zone and the crystalline zone $\Delta T<0$ ) was obtained to reduce the number of nuclei on the tungsten substrate. During growth, the proper positive values of $\Delta T \mathrm{~T}$ were chosen to content the supersaturation values $(0.25<\mathrm{S}<0.3)$. The reverse temperature condition during high temperature $\left(>1850^{\circ} \mathrm{C}\right)$ cooling was fulfilled to avoid recrystallization on grown AlN crystal. AlN single crystals made through the method were characterized by X-ray diffractions (XRD) and Raman spectroscopy.

Keywords: growth from vapour, single-crystal growth, nitrides, X-ray diffraction.
\end{abstract}

\section{INTRODUCTION}

Aluminium nitride (AlN) crystal is a typical representative of the third generation semiconductor materials. It possesses excellent electrical and optical properties such as high breakdown field strength, high thermal conductivity, and high carrier saturation velocity etc. Furthermore, AlN has an extra wide direct bandgap $(6.2 \mathrm{eV})$. AlN presents great potential for the application in deep-ultraviolet (DUV) optoelectronic devices such as light emitting diodes (LEDs), laser diodes (LDs) and photodetectors. Thus, AlN has attracted increasing attentions in the field of electronics and optoelectronics. In 2006, Taniyasu et al fabricated successfully AlN-based LEDs, which had a short wavelength about 210 nanometers $[1,2]$. AlN crystal can change from solid to liquid at high temperature $(\sim 3000 \mathrm{~K})$ and high vapor pressure $(\sim 200 \mathrm{~atm})$, so it is rarely fabricated from melt. Over the past several decades, it is proved that the physical vapor transport (PVT) is the most widely used and promising method of large-size single crystal AlN [3-7]. Different research groups studying AlN crystal growth have developed PVT process technologies. As of the publication date, a single AlN crystal of 1 inch in diameter has been grown by PVT [5-7]. The growth temperature for AlN crystals is so high that tungsten has been identified as an appropriate material for crucibles and substrate in which to grow AlN crystals. But the tungsten substrates are usually polycrystals. Usually, the sublimation temperature is higher than the crystallization temperature at the heating-up stage, which means $\Delta T>0$ (where $\Delta T$ is the temperature difference between sublimation zone and crystallization zone). There are high-density AlN

\footnotetext{
* Corresponding author. Tel.: +86-755-26538557; fax: +86-75526538557. E-mail address: hlwu@szu.edu.cn (H. Wu)
}

nucleation points on polycrystal tungsten substrates. Usually, we can only obtain polycrystalline AlN boules (e.g., AlN shown as Fig. 1 a).

In this study, the improved resistively-heated furnace will provide a vertical thermal gradient to reduce the number of nuclei on the substrate. At the heating-up stage, the value of $\Delta T$ is less than zero at the heating-up stage, which is called the condition of the reverse temperature field. During growth, the positive temperature gradient $(\Delta T>0)$ is established and close to thermal equilibrium growth conditions. Finally, we grow only a few detached AlN single crystals on the polycrystaline tungsten substrate.

\section{EXPERIMENTAL METHODS}

In general, PVT crystal-growth processes are performed in a graphite or tungsten heating element furnace with a resistive or inductive heating system, as in the case of SiC crystal growth by PVT. Due to its success in $\mathrm{SiC}$ crystal growth, the induction heating furnace has also been chosen for AlN growth $[8,9]$. In spite of the advantages of fast heating and energy savings, the induction heating furnace has the problems of carbon doping as well as the precise control of the temperature. In a purity nitrogen environment $(99.999 \%)$ at $0.8-1.2 \mathrm{~atm}$, the growth experiments were performed in a improved resistance-heated furnace. The growth unit (Fig. 2 c) has two heaters (main/top) and their respective infrared twocolor thermometers for temperature measurement. By adjusting the heaters which provide the sublimation zone and the crystalline zone with heat respectively, we can control the vertical temperature gradient accurately. And a tungsten crucible is employed to grow crystals. AlN polycrystals are used as the source and resublimated to achieve the high-purify AlN before growth. With this two- 
heater configuration, AIN can be fabricated on the nucleation zone, where conditions approach thermal equilibrium, producing bulk single crystals.

With an X-ray Diffraction (XRD) with $\mathrm{Cu} \mathrm{K} \alpha$ radiation on a Philips X-ray diffractometer at $40 \mathrm{kV}$ and $40 \mathrm{~mA}$, the crystal orientation and crystallinity of the samples were evaluated. Raman measurements were also employed via a Renishaw Raman spectrometer (inVia Reflex) with spectral resolution of $1.5 \mathrm{~cm}^{-1}$, where a $532 \mathrm{~nm}$ laser (Samba CW DPSS) with a power of $3 \mathrm{~mW}$ for exciting light was used.

\section{RESULTS AND DISCUSSION}

The relevant curves obtained by experimental and calculated results, which mainly relate to growth temperature $\left(>1850^{\circ} \mathrm{C}\right)$ and vapor pressure $(0.8-1.2 \mathrm{~atm})$ have described well the sublimation performance of AlN $[3,10]$. The equation $\mathrm{AlN}(\mathrm{s}) \leftrightarrow \mathrm{Al}(\mathrm{g})+1 / 2 \mathrm{~N}_{2}(\mathrm{~g})$ can indicate the process of AIN's sublimation-recondensation. Actually, there are five sub-procedures during AlN growth by PVT: $a$-sublimation of the source; $b$-mass transfer of the vapor species; $\mathrm{c}$-adsorption on the substrate surface; $\mathrm{d}$-diffusion and nucleation on the substrate surface, and $\mathrm{e}$-desorption [8]. We need low nucleation densities to grow high-quality bulk AIN crystal and a speed high enough for commercial production. The parameters can be optimized by simulations about the temperature field and supersaturation. In thermal equilibrium, the local supersaturation can be described by [11]

$S=\frac{p_{A l}^{2} \cdot p_{N_{2}}}{K(T)}-1$,

where $p_{A l}$ is the pressure of $\mathrm{Al}$ vapor, $p_{N 2}$ is the pressure of $\mathrm{N}_{2}$, and $K(T)$ is the equilibrium constant. Al vapour pressure $\left(p_{A l}\right)$ increases with temperature. When the ambient pressure $\left(p_{N 2}\right)$ is fixed in a given growth setup, alterable technological parameters are growth temperature $T$ and $\Delta T$. Excessive low supersaturation results in too low growth rates. On the contrary, exorbitant supersaturation leads to defective densities and high-density nucleation points that fabricate polycrystalline AlN. Many experiments with different $T$ and $\Delta T$ were carried out to investigate the adequate supersaturation range [7, 8, 11]. It has been proven that detached freestanding high-quality AlN single crystals with large size can be obtained when the values of supersaturation are between 0.25 and 0.3 [8]. The appropriate temperature for the AlN source and the nucleation zone ranges from $2050{ }^{\circ} \mathrm{C}$ to $2350{ }^{\circ} \mathrm{C}$ [5-7] which supplies sufficient surface mobility and mass transport for growth rates greater than or equal to $100 \mu \mathrm{m} / \mathrm{h}$. Due to the high vapor pressures of $\mathrm{Al}$ and $\mathrm{N}_{2}$ at these temperatures, the temperature gradients must be maintained at $5-20{ }^{\circ} \mathrm{C} / \mathrm{cm}$. The absence of the seeds makes researchers fabricate AlN polycrystals by spontaneous crystallization on planar W (or TaC) lids. In general, the AlN polycrystals are formed on the planar lid. To solve this problem, Slack et al designed a conical zone as the nucleation region in a tungsten crucible, shown in Fig. 2 a. The tip of the conical crucible allows a single crystal to grow in a dominant position [12]. But each crucible can only be used once and AlN single crystals above 1 inch in diameter can rarely be grown. Hartmann et al use the method of spontaneous nucleation and subsequent freestanding growth [6]. The growth setup (shown as Fig. 2 b), where a perforated sheet inside the crucible above the source material acts as a nucleation area, has been improved to promote spontaneous nucleation of large, high-quality AIN seeds. In this configuration, AIN can be grown on both the nucleation area, where conditions approach thermal equilibrium, and on the top of the tungsten crucible, typically as polycrystals. Now, this method only yields AlN single crystals about $10 \mathrm{~mm}$ in diameter.

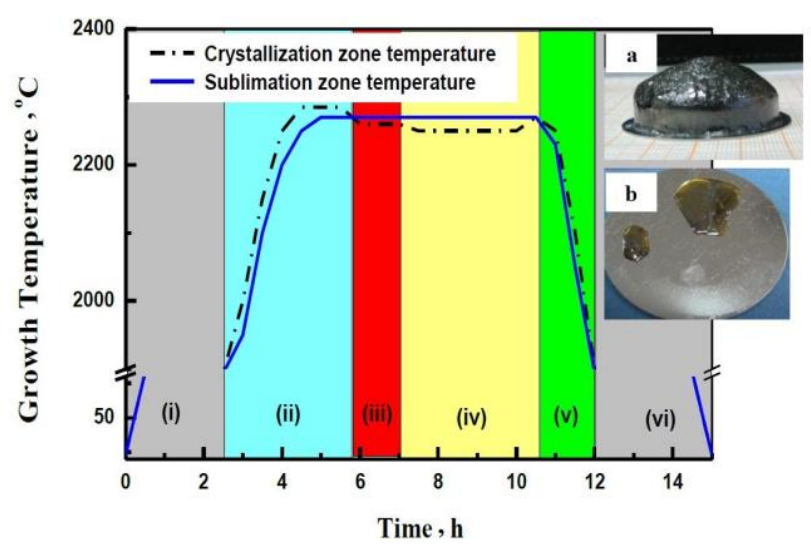

Fig. 1. Temperature variation trends of the crucible and the substrate during the growth process of AlN (i.e., from stage (i) to stage (vi)). The black line with dash dots represents the temperature of the crystallization zone and the blue line represents the temperature of the sublimation zone. The inset $a$ shows AlN crystals on the polycrystal tungsten substrates: $\mathrm{a}$-traditional growth model; $\mathrm{b}-\mathrm{a}$ reverse temperature field method

Generally, the temperature of sublimation zone is usually lower than that of crystallization zone (i.e., $\Delta T>0$ ) during the whole process. In this work, the AlN crystal growth can be divided into six stages: (i) low temperature pre-heating, (ii) high temperature pre-heating, (iii) nucleation, (iv) growth, (v) high temperature cooling and (vi) low temperature cooling. Fig. 1 shows the temperature variation trends of the crucible and the substrate during the growth process of AlN. During the stage (i) and (vi), there are no special requirement for the value of $\Delta T$ because the temperature is lower than the temperature of $\mathrm{AlN}$ sublimation $\left(\sim 1850{ }^{\circ} \mathrm{C}\right)$. While the temperature is above $1850{ }^{\circ} \mathrm{C}$ on the stage (ii) (blue area in Fig. 1), there is a reverse temperature field (i.e., $\Delta T<0$ ), which makes it impossible for AlN nucleation on the substrate. The growth enters the nucleation stage (iii) (red area in Fig. 1) by reducing crystallization temperature after reaching the highest sublimation temperature. During this stage, maintaining the appropriate $\Delta T$ will help to reduce the number of nuclei on the tungsten substrate, where growth conditions is approximate to thermal equilibrium. To refer to the preceding supersaturation values $(0.25<\mathrm{S}<0.3)$ on the stage (iv) (green area in Fig. 1), several divided AlN single crystals will be obtained on the substrate (shown as Fig. $1 \mathrm{~b}$ ). According to the experimental data, we also simulated the temperature field on the stage (iii) and (iv). 

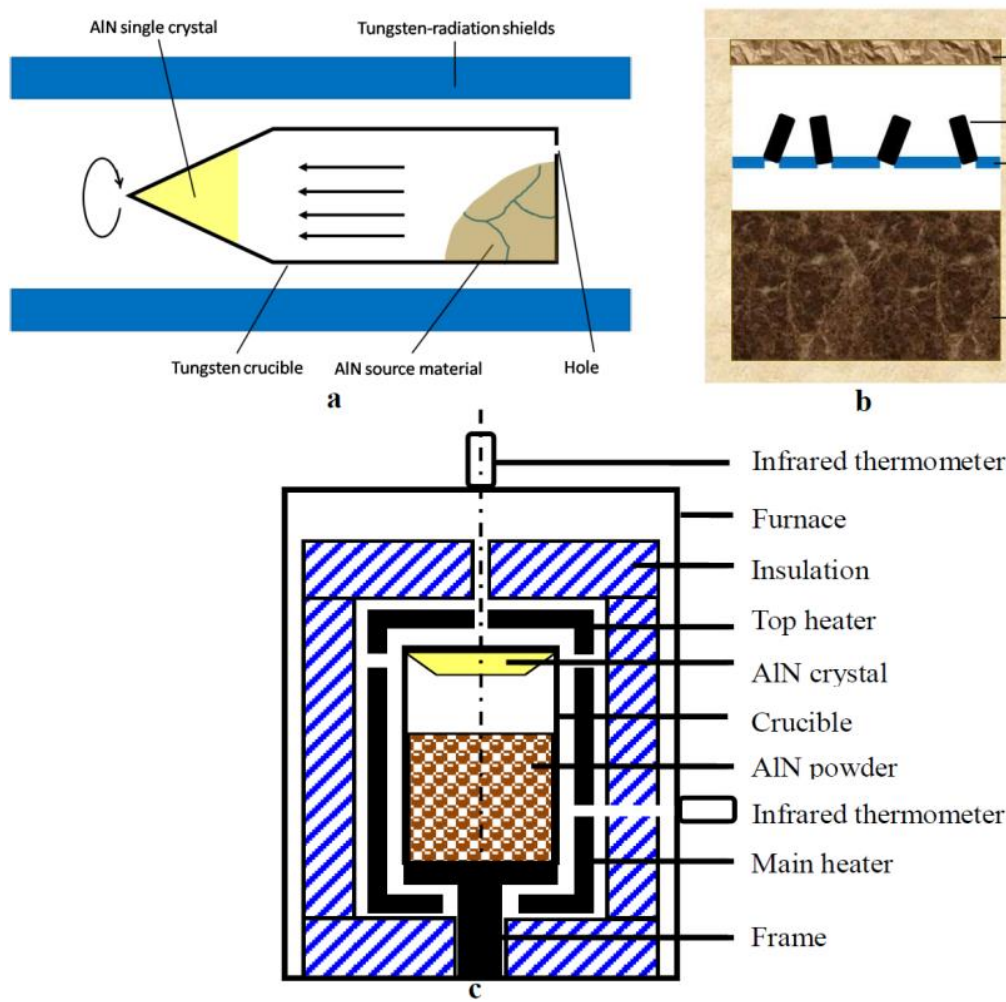

Infrared thermometer

Furnace

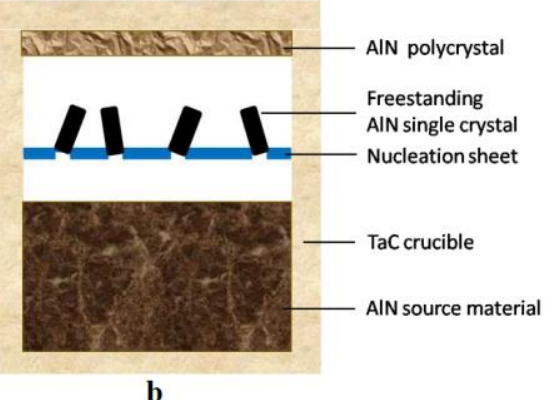

.

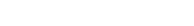


positions of the four modes are centered at $620.1 \mathrm{~cm}^{-1}$, $656.8 \mathrm{~cm}^{-1}, 669.3 \mathrm{~cm}^{-1}, 913.1 \mathrm{~cm}^{-1}$, respectively, which agree well with other reports $[13,14]$. The full-width at half maximum (FWHM) of $\mathrm{E}_{2}^{2}$ mode is less than $8.6 \mathrm{~cm}^{-1}$, indicating that the AIN crystal quality is high.

\section{CONCLUSIONS}

In this manuscript, the improved resistively-heated furnace is employed to nucleation control in PVT growth of AlN single crystals on polycrystal tungsten substrates. The growth unit includes two heaters for a vertical temperature gradient between the sublimation zone and the crystalline zone. The reverse temperature field (i.e., $\Delta T<0)$ during high temperature $\left(>1850{ }^{\circ} \mathrm{C}\right)$ heating and high temperature cooling, can help avoid nucleation on the substrate and recrystallize on AlN crystal, respectively. During the growth stage, $\Delta T$ are chose to the proper positive values, which make growth conditions approach thermal equilibrium states. Finally, only several separated AlN single crystals have been prepared.

\section{Acknowledgments}

The authors gratefully acknowledge the financial support from the National Natural Science Foundation of China (Grant No. 61974094, 61440028 and 61136001) and the Science \& Technology Bureau of Shenzhen (Grant No. 20160520174438578).

\section{REFERENCES}

1. Taniyasu, Y., Kasu, M., Makimoto, T. An Aluminium Nitride Light-emitting Diode with a Wavelength of 210 Nanometres Nature 441 (7091) 2006: pp. 325-328. https://doi.org/10.1038/nature04760

2. Taniyasu, Y., Kasu, M., Makimoto, T. Aluminum Nitride Deep-ultraviolet Light-emitting p-n Junction Diodes Diamond and Related Materials $17(7-10)$ 2008: pp. $1273-1277$. https://doi.org/10.1016/j.diamond.2008.02.042

3. Slack, G.A., McNelly, T.F. Growth of High Purity AlN Crystals Journal of Crystal Growth 34 (2) 1976: pp. $263-279$. https://doi.org/10.1016/0022-0248(76)90139-1

4. Singh, N.B., Berghmans, A., Zhang, H., Wait, T., Clarke, R.C., Zingaro, J., Golombeck, J.C. Physical Vapor Transport Growth of Large AlN Crystals Journal of Crystal Growth 250 (1) 2003: pp. 107-112. https://doi.org/10.1016/S0022-0248(02)02235-2

5. Raghothamachar, B., Dalmau, R., Moody, B., Craft, S., Schlesser, R., Xie, J., Collazo, R., Dudley, M., Sitar, Z. Low Defect Density Bulk AlN Substrates for High
Performance Electronics and Optoelectronics

Materials Science Forum 717 2012: pp. 1287-1290.

https://doi.org/10.4028/www.scientific.net/MSF.717720.1287

6. Hartmann, C., Dittmar, A., Wollweber, J., Bickermann, M. Bulk AlN Growth by Physical Vapour Transport Semiconductor Science \& Technology 29 (8) 2014: pp. 084002.1-084002.10. https://doi.org/10.1088/0268-1242/29/8/084002

7. Liu, G., Zhou, G., Qin, Z., Zhou, Q., Zheng, R., Wu, H., Sun, Z. Luminescence Characterizations of Freestanding Bulk Single Crystalline Aluminum Nitride towards Optoelectronic Application CrystEngComm 19 (37) 2017: pp. $5522-5527$. http://dx.doi.org/10.1039/C7CE01239J

8. Lu, P., Collazo, R., Dalmau, R.F., Durkaya, G., Dietz, N., Raghothamachar, B., Dudley, M., Sitar, Z. Seeded Growth of AlN Bulk Crystals in $\mathrm{m}$ - and c-orientation Journal of Crystal Growth 312 (1) 2009: pp. 58-63. https://doi.org/10.1016/j.jcrysgro.2009.10.008

9. Wu, B., Zhang, H. Transport Phenomena in an Aluminum Nitride Induction Heating Sublimation Growth System International Journal of Heat and Mass Transfer 47 (14-16) 2004: pp. 2989-3001. https://doi.org/10.1016/j.ijheatmasstransfer.2004.03.001

10. Epelbaum, B.M., Nagata, S., Bickermann, M., Heimann, P., Winnacker, A. Development of Natural Habit of Large Free-nucleated AIN Single Crystals Physica Status Solidi (b) 244 (6) 2007: pp. 1780-1783. https://doi.org/10.1002/pssb.200674835

11. Hartmann, C., Wollweber, J., Dittmar, A., Irmscher, K., Kwasniewski, A., Langhans, F., Neugut, T., Bickermann, M. Preparation of Bulk AlN Seeds by Spontaneous Nucleation of Freestanding Crystals Japanese Journal of Applied Physics 52 (8) 2013: pp. 279-287. https://doi.org/10.7567/JJAP.52.08JA06

12. Schowalter, L.J., Slack, G.A., Whitlock, J.B., Morgan, K., Schujman, S.B., Raghothamachar, B., Dudley, M., Evans, K. Fabrication of Native, Single-crystal AlN Substrates Physica Status Solidi(c) 7 2003: pp. $1997-2000$. https://doi.org/10.1002/pssc.200303462

13. Pandit, P., Song, D.Y., Holtz, M. Decay of Zone-center Phonons in AlN with A1, E1, and E2 Symmetries Journal of Applied Physics $102(11)$ 2007: pp. 113510.1-113510.4. https://doi.org/10.1063/1.2821360

14. Song, D.Y., Holtz, M., Chandolu, A., Nikishin, S., Mokhov, E., Makarov, Y., Helava, H. Optical Phonon Decay in Bulk Aluminum Nitride Applied Physics Letters 89 (2) 2006: pp. 021901.1-021901.3. https://doi.org/ 10.1063/1.2219092 